\title{
R-D OPTIMIZED AUXILIARY INFORMATION FOR INPAINTING-BASED VIEW SYNTHESIS
}

\section{Ismael Daribo, Gene Cheung}

\author{
National Institute of Informatics (NII) \\ Tokyo, Japan
}

\author{
Thomas Maugey, Pascal Frossard
}

\author{
Signal Processing Laboratory (LTS4) \\ Ecole Polytechnique Fédérale de Lausanne (EPFL) \\ Lausanne, Switzerland
}

\begin{abstract}
Texture and depth maps of two neighboring camera viewpoints are typically required for synthesis of an intermediate virtual view via depth-image-based rendering (DIBR). However, the bitrate overhead required for reconstruction of multiple texture and depth maps at decoder can be large. The performance of multiview video encoders such as MVC is limited by the simple fact that the chosen representation is inherently redundant: a texture or depth pixel visible from both camera viewpoints is represented twice. In this paper, we propose an alternative 3D scene representation without such redundancy, yet at decoder, one can still reconstruct texture and depth maps of two camera viewpoints for DIBR-based synthesis of intermediate views. In particular, we propose to first encode texture and depth videos of a single viewpoint, which are used to synthesize the uncoded viewpoint via DIBR at decoder. Then, we encode additional rate-distortion (RD) optimal auxiliary information (AI) to guide an inpainting-based hole-filling algorithm at decoder and complete the missing information due to disocclusion. For a missing pixel patch in the synthesized view, the AI can: i) be skipped and then let the decoder by itself retrieve the missing information, ii) identify a suitable spatial region in the reconstructed view for patch-matching, or iii) explicitly encode missing pixel patch if no satisfactory patch can be found in the reconstructed view. Experimental results show that our alternative representation can achieve up to $41 \%$ bit-savings compared to H.264/MVC implementation.
\end{abstract}

Index Terms - Texture-plus-depth format, depth-image-based rendering, compact representation

\section{INTRODUCTION}

In recent years, the interest in $3 \mathrm{D}$ video communication end-toend services is constantly increasing and has led to interactivity and 3D perception improvements in related applications, including Three-Dimensional Television (3D-TV) and Free Viewpoint Television (FTV). This breakthrough has been promoted by the recent development of auto-stereoscopic displays, multi-cameracaptured systems and depth acquisition technologies. The potential benefit of capturing a scene from different viewpoints through Multiview Video (MVV) communication system, has gained recent attention. Despite a limited continuum of views, MVV communication systems can provide user navigation with a look-around sensation by view synthesis via depth-image-based rendering (DIBR) at decoder [1]. Views that are not captured from a real camera can be synthesized using texture and depth maps of two neighboring

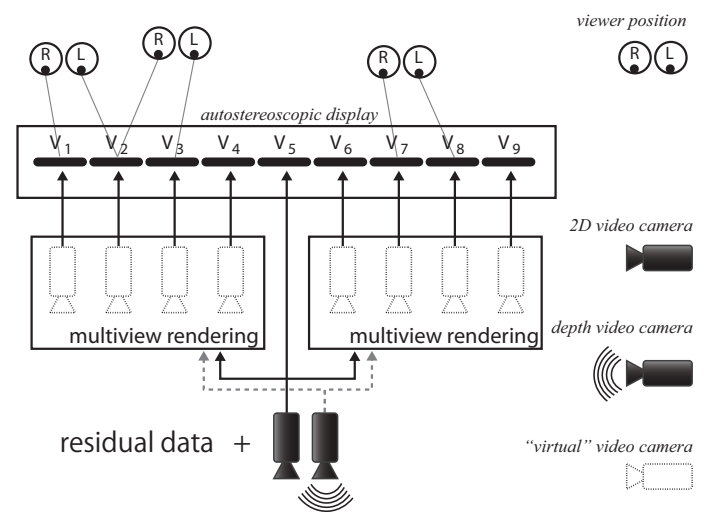

Figure 1. Proposed interactive multiview imaging system at decoder. Virtual views are synthesized around the camera-captured view by DIBR assisted with residual data.

camera-captured views. With DIBR-based view synthesis, only texture and depth videos of a subset of anchor views are needed at decoder for reconstruction of all intermediate virtual views used for smooth view transition.

To reduce the bitrate overhead required for the reconstruction of multiple texture videos at decoder, multiview video coding (MVC) schemes [2] encode them for multiple viewpoints using disparity compensation to exploit cross-view correlation. While MVC has shown coding gain over more naïve independent view coding approaches, its performance is limited by the simple fact that the chosen representation is inherently redundant ${ }^{1}$ : namely, a texture or depth pixel visible from two reference views is represented twice.

In this paper, we propose an alternative 3D scene representation without pixel redundancy, yet by encoding enough information for DIBR-based synthesis of intermediate views at decoder. In particular, we propose to first encode texture and depth video of a single viewpoint, which are used to synthesize the other uncoded viewpoint via DIBR at decoder. Then, motivated by our earlier work [3], we design auxiliary information (AI) that is used to guide an inpainting-based hole-filling ${ }^{2}$ algorithm [5] at decoder. The key idea is that non-local but correlated pixel patches in the reconstructed image can be maximally exploited to

\footnotetext{
${ }^{1}$ For reference viewpoints that are physically close to each other, the intensity difference of a pixel viewed from different viewpoints for most objects is likely small. Further, it is not clear if encoding the pixel difference contributes to view synthesis quality during pixel blending.

${ }^{2}$ Unlike typical 2D image inpainting scenarios, partial 3D geometric information (depth map) can be exploited during pixel-filling [4].
} 
complete missing pixels due to disocclusion. Then we propose to selectively encode the additional information in a rate-distortion (RD) optimal way. Specifically, for a given missing pixel patch in the synthesized view, the AI can: i) be skipped and let the decoder by itself retrieve the missing information, ii) identify a suitable spatial region in the reconstructed view for patch-matching, or iii) explicitly encode missing pixel patch if no satisfactory patch can be found in the reconstructed view. Experimental results show that our alternative representation can reduced bitrate by up to $41 \%$ compared to MVC for the same synthesized view quality.

The outline of the paper is as follows. We first discuss related work in Section 2. We then overview our interactive multiview video system in Section 3. We discuss how AI are designed and selected in an RD-optimal manner in Section 4. Finally, experimental results and conclusions are presented in Section 5 and 6 , respectively.

\section{RELATED WORK}

From a representation perspective, the most related work in the literature is the layered depth video (LDV) representation [6], where texture and depth maps of a single viewpoint is first encoded as the main layer, then occluded spatial regions in other camera viewpoints are added as enhancement layers. We first note that LDV, like our proposed representation, also avoids the pixel representation redundancy problem in MVC. However, we differ from LDV in the following aspects. First, we use a hole-filling algorithm ${ }^{3}$ to complete missing pixels in the projected anchor view, while LDV typically used traditional coding tools based on transform plus entropy coding to explicitly encode disoccluded regions. Second, we design and employ RD-optimal AI to guide the hole-filling algorithm to further improve quality of the synthesized reference view. In the experimental section, we will show the performance gain of our scheme against LDV.

From a methodology perspective, the most similar work is an image compression algorithm in [8], where assistant information (edges in a code block) was encoded to aid a decoder edge-based inpainting scheme to reconstruct missing blocks. Though similar in spirit to our proposed AI, our proposal differs in the following aspect. First, our AI can provide location information to guide a non-local exemplar-based hole-filling algorithm to a spatial region with similar textural patches. In contrast, assistant information in [8] provides only edge information, which is used only for a local structural inpainting method that uses prior assumptions about the smoothness of the structures in the missing regions to propagate boundary data. It has been shown that non-local textural exemplar-based inpainting methods [5] often outperform local structural methods when the smoothness assumption is no more valid. Second, unlike block-based image coding, a disoccluded patch can be of arbitrary shape, so in the case when it is not possible for a hole-filling algorithm to locate a satisfactory similar patch, we efficiently encode the arbitrarily shaped pixel patch using the Graph-Based Transform (GBT) [9].

\section{PROPOSED SYSTEM}

\subsection{Encoder/Decoder Communication}

In the proposed interactive multiview communication system, a user freely navigates among anchor views with smooth transition.

\footnotetext{
${ }^{3}$ In our earlier work [7], an inpainting algorithm was used in a straightforward manner for hole-filing in the projected view, but no RD-optimal AI was designed and deployed to enhance the quality of the inpainted patches.
}

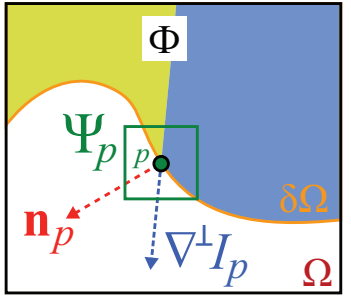

Figure 2. Notation diagram of an exemplar-based inpainting technique (from [5]). The current patch $\Psi_{p}$ to be filled in, centered at the point $p$ on the boundary $\delta \Omega$, is overlaying over the missing region $\Omega$ and the source region $\Phi$.

If the requested view is itself an reference view, the full view is explicitly transmitted. Otherwise, the user is first given the closest reference view (color and depth videos). Then, RD-optimal AI are additionally transmitted by encoder, so that another reference view-one where the requested virtual view becomes an intermediate view between the two references - can be constructed via DIBR using texture and depth maps of the first reference, plus inpainting guided by the transmitted AI. The desired virtual view is finally synthesized via DIBR using the two constructed reference views. It is important to note that the proposed RD optimization of the $\mathrm{AI}$ at encoder, is reference-view-dependent and, thus, does not depend on particular virtual views synthesized at decoder. Let us consider the current reference view $V_{i}$ and the next closest reference view $V_{j}$. AI is RD-optimized for all occluded spatial regions in reference view $V_{i}$ but visible in reference view $V_{j}$.

In the next section, let us review the hole-filling method based on the well-known Criminisi's algorithm [5]. Though we chose this specific implementation of exemplar-based techniques for concreteness, it is important to note that our proposed optimization framework extends beyond this specific scheme.

\subsection{Inpainting-based Hole-Filling}

Due to the natural similarity between damaged spatial regions in paintings and disocclusions in view synthesis, one solution consists in combining IBR with inpainting techniques to deal with disocclusions. Criminisi et al. [5] first reported that exemplarbased texture synthesis contains the process necessary to replicate both texture and structure.

With input image $I$ and missing region $\Omega$, the source region $\Phi$ is defined as $\Phi=I-\Omega$, and the boundary of the missing region is indicated by $\delta \Omega$ as illustrated in Fig. 2. For every patch $\Psi_{p}$ centered at the point $p$, where $p \in \delta \Omega$, the patch $\Psi_{p}$ can be decomposed into two disjoint sub-regions such that

$$
\Psi_{p}=\left(\Psi_{p} \cap \Phi\right) \cup\left(\Psi_{p} \cap \Omega\right) \quad \text { and } \quad \emptyset=\left(\Psi_{p} \cap \Phi\right) \cap\left(\Psi_{p} \cap \Omega\right)
$$

where both $\Psi_{p} \cap \Phi$ and $\Psi_{p} \cap \Omega$ are known at the encoder, while the decoder only has knowledge of $\Psi_{p} \cap \Phi$.

\subsubsection{Priority computation}

It has been shown that the quality of the output image synthesis is greatly influenced by the order in which the inpainting is processed [5]. In addition, in the context of DIBR system, disocclusions are the result of displaced foreground object that reveals some background areas. Filling in the disoccluded regions using background pixels therefore makes more sense than foreground ones [4]. More priority is then given to patches that overlay regions where the depth variance is low, excluding regions at the 
foreground/background boundaries. The selection of the current patch to be filled in can be formulated as

$$
\Psi_{p^{*}}=\underset{p \in \delta \Omega}{\arg \max }\left\{C\left(\Psi_{p}\right) \cdot D\left(\Psi_{p}\right) \cdot L\left(\Psi_{p}\right)\right\},
$$

where $C$ is the confidence term that indicates the reliability of the current patch, $D$ is the data term that gives special priority to the isophote direction, and $L$ is the level regularity term as the inverse square variance of the depth patch. For the sake of brevity, we will not describe the different terms: for more details, the reader is referred to [4].

\subsubsection{Patch matching}

As originally defined by Criminisi et al. [5], once the highest priority patch $\Psi_{p^{*}}$ is selected, a block matching algorithm derives the best exemplar patch $\Psi_{q^{*}}$ to fill in the missing pixels under the patch $\Psi_{p^{*}}$ such that

$$
\Psi_{q^{*}}=\underset{q \in \Phi}{\arg \min }\left\{d\left(\Psi_{p^{*}} \cap \Phi, \Psi_{q} \cap \Phi\right)\right\}
$$

where the distance $d(.,$.$) is defined as the Sum of Squared Differ-$ ences (SSD).

Having found the source exemplar $\Psi_{q^{*}}$, the value of each pixel-to-be-filled $p^{\prime} \in \Psi_{p^{*}} \cap \Omega$ is copied from its corresponding pixel in $\Psi_{q^{*}}$. After the patch $\Psi_{p^{*}}$ has been filled, the confidence term $\mathrm{C}(\mathrm{p})$ is updated as follows

$$
C(p)=1 \quad \forall p \in \Psi_{p^{*}} \cap \Omega .
$$

\section{DESIGN OF AUXILIARY INFORMATION}

\subsection{Types of AI}

The solution of Eq. (3) can diverge, however. This is due to the fact that the minimization is done only on the sub-region $\Psi_{p^{*}} \cap \Phi$. To tackle this issue, we propose to assist the inpainting process with AI that prevents the aforementioned solution divergence. The proposed framework supports four different $\mathrm{AI} \varphi_{p}$, where $\varphi_{p} \in$ $\left\{\varphi_{\text {skip }}, \varphi_{\text {intra }}, \varphi_{\text {pred }}, \varphi_{\text {mv }}\right\}$ such that

- $\varphi_{\text {skip }} \equiv$ no information is sent. As a result, at the decoder side, the patch is classically inpainted by minimization of the distance function over the source sub-region $\Psi_{p^{*}} \cap \Phi$ as expressed in Eq. (3),

- $\varphi_{\text {intra }} \equiv$ the quantized transformed coefficients of the decoderside-missing-regions $\Psi_{p} \cap \Omega$ are explicitly delivered directly to the decoder such that

$$
\varphi_{\text {intra }}:=Q\left(\zeta\left(\Psi_{p} \cap \Omega\right)\right)
$$

where the transform domain function $\zeta$ represents the GraphBased Transform (GBT) [9], which fits well the arbitrarily shaped region $\Psi_{p} \cap \Omega$. $Q$ being an uniform quantization function,

- $\varphi_{\text {pred }} \equiv$ after inpainted prediction, such that the inpainting process at the decoder side is reproduced at the encoder, the quantized transformed coefficients of the remaining residual is sent as follows

$$
\varphi_{\text {pred }}:=Q\left(\zeta\left(\Psi_{\text {res }} \cap \Omega\right)\right), \quad \text { with } \quad \Psi_{\text {res }}=\Psi_{p}-\Psi_{q^{*}}
$$

where

$$
\Psi_{q^{*}}=\underset{q \in \Phi}{\arg \min } d\left(\Psi_{p} \cap \Phi, \Psi_{q} \cap \Phi\right)
$$

where the distance $d(.,$.$) is defined as the Sum of Squared$ Differences (SSD),
- $\varphi_{\mathrm{mv}} \equiv$ in a more traditional way, the ground truth is fully utilized to compute the motion vector $\mathbf{m v}$ that minimize the Lagrangian function cost such that $\varphi_{\mathrm{mv}}:=\mathbf{m v}^{*}$ with

$$
\mathbf{m v}^{*}=\underset{p+\mathbf{m v} \in \Phi}{\arg \min }\left\{d\left(\Psi_{p}, \Psi_{p+\mathbf{m v}}\right)+\lambda \cdot \mathbf{R}(\mathbf{m v})\right\},
$$

where all possible motion vectors are restrained within a search window.

At decoder side, we then propose to modify Eq. (3) to support the proposed AI as follows

$$
\Psi_{q^{*}}= \begin{cases}\Psi_{q^{*}}^{0} & \text { if } \varphi_{p^{*}}=\varphi_{\text {skip }} \\ \zeta^{-1}\left(Q^{-1}\left(\varphi_{\text {intra }}\right)\right) & \text { if } \varphi_{p^{*}}=\varphi_{\text {intra }} \\ \Psi_{q^{*}}^{0}+\zeta^{-1}\left(Q^{-1}\left(\varphi_{\text {pred }}\right)\right) & \text { if } \varphi_{p^{*}}=\varphi_{\text {pred }} \\ \Psi_{p^{*}+\varphi_{\mathrm{mv}}} & \text { if } \varphi_{p^{*}}=\varphi_{\mathrm{mv}}\end{cases}
$$

where the functions $\zeta^{-1}$ and $Q^{-1}$ is the inverse GBT and quantization function, respectively. $\Psi_{q^{*}}^{0}$ being defined in Eq.(3), represents the selected patch in a traditional inpainting algorithm, i.e., no $\mathrm{AI}$ is utilized.

\subsection{RD Optimized Coding of AI}

Given a delivered AI represented by $\varphi=\left\{\varphi_{p}\right\}$, we propose to re-formulate the hole-filling problem in an RD manner as follows

$$
\underset{\Psi_{p}}{\arg \min } \int_{\delta \Omega}\left(\operatorname{SSD}\left(\Psi_{p} \cap \Omega \mid \varphi_{p}\right)+\lambda \cdot \mathrm{R}\left(\varphi_{p}\right)\right) \mathrm{d} p
$$

where at the location $p$ the SSD measurement quantifies an estimate of the inpainted reconstructed quality of the missing regions, while $\mathrm{R}$ measures the bits needed to encode the $\mathrm{AI} \varphi_{p}$ that assists the inpainting process. Here, $\lambda \geq 0$ is the Lagrangian multiplier.

Under the assumption that both encoder and decoder are using the same inpainting algorithm, it is possible to RD optimize the $\mathrm{AI} \varphi$ to deliver to the decoder, which improves the overall reconstruction quality as described in Eq. (6). For a given quantization parameter $q p$, finding the optimal RD-driven $\mathrm{AI} \varphi$ can be formulated through the minimization of the following Lagrangian criterion:

$$
\underset{\varphi=\left\{\varphi_{p}\right\}}{\arg \min } \int_{\delta \Omega}\left(\operatorname{SSD}\left(\Psi_{p}, \varphi_{p} \mid q p\right)+\lambda \cdot \mathrm{R}\left(\varphi_{p} \mid q p\right)\right) \mathrm{d} p
$$

with $\lambda$ as defined in H.264 standard

$$
\lambda=0.85 \cdot 2^{q p-12} \cdot 4
$$

In addition, it is important to note that the ground truth of the missing-regions $\Omega$ is known at the encoder side.

\section{EXPERIMENTAL RESULTS}

The performance of the proposed framework was evaluated using the multiview video dataset Ballet and Breakdancers (1024×768@15 Hz) provided by Microsoft In the experiments, the camera 4 is used as anchor view, and the view 5 as synthesized one. The comparison of objective compression performance is illustrated in the rate-distortion (RD) curves plotted in Fig. 3, where the peak signal-to-noise ratio (PSNR) of the synthesized texture video is plotted against bitrate (kbits/frame) over 100 frames. The $\mathrm{RD}$ results correspond to five $q p$ quantization parameters: 24 , 


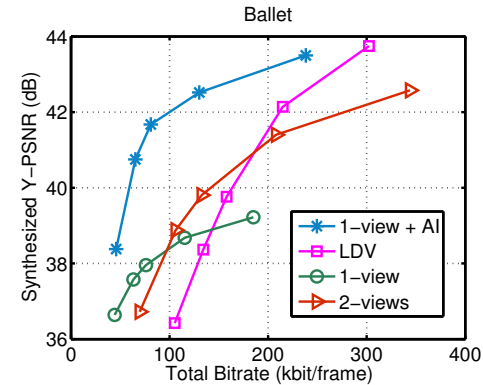

(a)

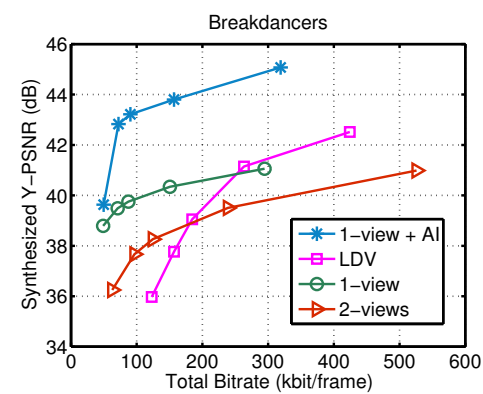

(b)

Figure 3. RD comparison of our proposed scheme "1-view+AI" against: LDV [6], "1-view" scheme where no AI is sent, and "2-views" scheme where two anchor views are sent.

$28,32,34$, and 38 . The bitrate consists of the sum of the anchor view rate plus the residual data rate. As shown in Fig. 3 we compare our proposed "1-view+AI" scheme against three others schemes: LDV [6], "1-view", "2-views". The proposed "1view+AI" scheme consists in encoding one anchor view and AI to assist the hole-filling at decoder, as described previously. LDV corresponds to the specific case of sending only INTRA AI. The "1-view" scheme consists in sending only the anchor view, which is equivalent to delivering no AI (i.e., SKIP mode). The "2-views" scheme consists of explicitly sending the two closest anchor views. We used the implementation of H.264/MVC standard JMVC 7.0, to exploit the cross-layer and inter-view correlation in the LDV and "2-views" representation, respectively.

We see that our new compact representation "1-view+AI" results in significant compression gain. Specifically, an average bitrate reduction up to $41 \%$ and $35 \%$ for the multiview dataset Ballet and Breakdancers, respectively, are observed. It can be also observed in Fig. 4 that the average distribution of the different $\mathrm{AI}$ at different quantization parameter $q p$. As expected, at low bitrate (i.e., high quantization parameter $q p$ ) the bitrate saving comes from the over selection of SKIP AI, while at high bitrate the motion vector AI gradually replaces the INTRA AI.

\section{CONCLUSION}

In this paper we proposed an alternate 3D scene representation without pixel redundancy. We first encode texture and depth videos of a single view as anchor view, which are used to synthesize a second reference view at decoder. Then, we encode RD-optimal additional auxiliary information (AI) to guide an inpainting-based hole-filling algorithm at decoder and complete missing information due to disocclusions. Experimental results show an overall bitrate reduction up to $41 \%$ over a classical H.264/MVC implementation.

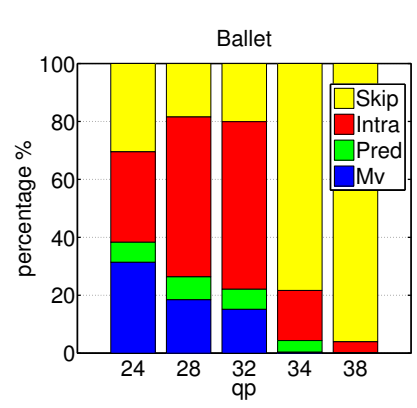

(a) Ballet

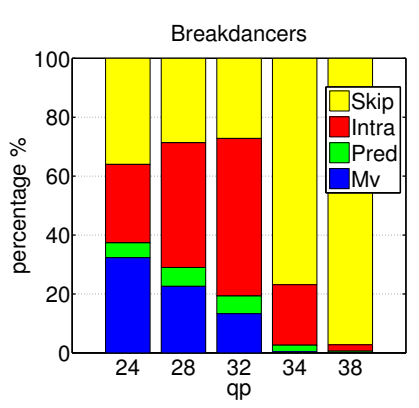

(b) Breakdancers
Figure 4. AI mode distribution for different values of the quantization parameter $q p$.

\section{Acknowledgment}

This work is partially supported by the JSPS Postdoctoral Program for Foreign Researchers in Japan, and the Swiss National Science Foundation under grant 200021_126894.

\section{REFERENCES}

[1] Liu Zhan-wei, An Ping, Liu Su-xing, and Zhang Zhao-yang, "Arbitrary view generation based on DIBR," in Proc. of the International Symposium on Intelligent Signal Processing and Communication Systems (ISPACS), 2007, pp. 168-171.

[2] P. Merkle, K. Müller, A. Smolic, and T. Wiegand, "Efficient compression of multi-view video exploiting inter-view dependencies based on H.264/MPEG4-AVC," in Proc. of the IEEE International Conference on Multimedia and Expo (ICME), 9-12 July 2006, pp. 1717-1720.

[3] T. Maugey, P. Frossard, and G. Cheung, "Consistent view synthesis in interactive multiview imaging," in Proc. of the IEEE International Conference on Image Processing (ICIP), Orlando, Florida, USA, Oct. 2012.

[4] I. Daribo and B. Pesquet-Popescu, "Depth-aided image inpainting for novel view synthesis," in Proc. of the IEEE Workshop on Multimedia Signal Processing (MMSP), oct. 2010, pp. $167-170$.

[5] A. Criminisi, P. Perez, and K. Toyama, "Region filling and object removal by exemplar-based image inpainting," IEEE Transactions on Image Processing, vol. 13, no. 9, pp. 12001212, 2004.

[6] K. Muller, A. Smolic, K. Dix, P. Kauff, and T. Wiegand, "Reliability-based generation and view synthesis in layered depth video," in Proc. of the IEEE Workshop on Multimedia Signal Processing (MMSP), Cairns, Queensland, Australia, Oct. 2008, pp. 34-39.

[7] I. Daribo and H. Saito, "A novel inpainting-based layered depth video for 3DTV," IEEE Transactions on Broadcasting, vol. 57, no. 2, pp. 533-541, june 2011.

[8] Dong Liu, Xiaoyan Sun, Feng Wu, Shipeng Li, and Ya-Qin Zhang, "Image compression with edge-based inpainting," IEEE Transactions on Circuits and Systems for Video Technology, vol. 17, no. 10, pp. 1273-1287, 2007.

[9] G. Shen, W.-S. Kim, S.K. Narang, A. Ortega, Jaejoon Lee, and Hocheon Wey, "Edge-adaptive transforms for efficient depth map coding," in Proc. of the Picture Coding Symposium (PCS), Nagoya, Japan, Dec. 2010, pp. 566-569. 